\title{
Una intervención basada en mensajes de texto fue útil para modificar factores de riesgo cardiovascular en pacientes con enfermedad coronaria
}

An intervention based on text messaging was useful to modify cardiovascular risk factors in patients with coronary heart disease

Chow C. y col. JAMA, 2015;314(12):1255-1263

\section{Objetivos}

Evaluar el efecto de una intervención basada en la entrega de mensajes de texto (SMS) para incentivar la modificación de factores de riesgo cardiovascular en pacientes con enfermedad coronaria.

\section{Diseño}

Ensayo clínico aleatorizado, simple ciego.

\section{Población y lugar}

Los participantes fueron reclutados de un centro de tercer nivel de atención, de carácter público, ubicado en una zona de bajos recursos de Sídney, Australia. Fueron seleccionadas personas con enfermedad coronaria (EC) establecida, definiéndose ésta última como episodio documentado de infarto agudo de miocardio (IAM), angioplastia o estenosis mayor o igual al $50 \%$ de un vaso epicárdico.

\section{Intervención}

Fueron asignados aleatoriamente 710 participantes a sendos grupos. El grupo intervención $(n=352)$ recibió los cuidados habituales más cuatro SMS semanales durante seis meses. El grupo control $(n=358)$ recibió solamente los cuidados habituales. Los SMS brindaban consejo, motivación e información sobre hábitos de vida saludables. Los mensajes eran personalizados en relación a las características basales de cada participante. No eran interactivos.

\section{Medición de resultados principales}

El resultado primario fue el valor de colesterol LDL a los seis meses. Fueron establecidos como resultados secundarios los niveles de presión arterial sistólica, la prevalencia de tabaquismo, la práctica de actividad física y el índice de masa corporal. El análisis de las variables se realizó por intención de tratar.

\section{Resultados}

Se resumen en la tabla 1.

Tabla 1: resultados de la intervención motivacional basada en mensajes de texto comparados con los del grupo control.

\begin{tabular}{l|c|c|c|c}
\multirow{2}{*}{\multicolumn{1}{c|}{ Resultado }} & \multicolumn{2}{|c|}{ Media (IC95\%)* por grupo } & \multirow{2}{*}{$\begin{array}{c}\text { Diferencia media } \\
\text { (IC95\%)* }\end{array}$} & p \\
\cline { 2 - 3 } Colesterol LDL (mg/dL) & Intervención & Control & $-5(-9 \mathrm{a} 0)$ & 0,04 \\
Presión arterial sistólica (mmHg) & $79(76 \mathrm{a} 82)$ & $84(81 \mathrm{a} 87)$ & $-7,6(-9,8 \mathrm{a}-5,4)$ & $<0,001$ \\
Índice de masa corporal & $128,2(126,7 \mathrm{a}$ 129,8) & $135,8(134,3 \mathrm{a} 137,3)$ & $-1,3(-1,6 \mathrm{a}-0,9)$ & $<0,001$ \\
Actividad física (MET min/sem) & $29(28,8 \mathrm{a} 29,3)$ & $30,3(30,1 \mathrm{a} 30,5)$ & $293,4(102 \mathrm{a} 484,8)$ & 0,003 \\
\hline Tabaquistas/Total (\%) & $936,1(69,6)$ & $642,7(68,1)$ & & $<0,001$ \\
\hline
\end{tabular}

${ }^{*}$ IC95\%: intervalo de confiaza del 95\%.

\section{Conclusiones}

En los pacientes con EC, y en comparación con el cuidado habitual, el uso de SMS para incentivar cambios de vida saludables, resultó en una modesta mejoría en los valores de colesterol LDL y una gran mejoría en los otros factores de riesgo cardiovascular analizados.

Fuente de financiamiento: National Heart Foundation of Australia Grant y BUPA Foundation.

\section{Comentario}

Este ensayo clínico aporta evidencia acerca de la modificación de múltiples factores de riesgo a partir de una intervención tecnológica de bajo costo. Estudios previos habían demostrado su eficacia en personas con diabetes ${ }^{1} \mathrm{y} / \mathrm{o}$ hipertensión ${ }^{2}$, pero este es uno de los primeros trabajos en desarrollar un sistema de SMS que podría abarcar varios factores de riesgo a la vez. Vale destacar que como el $100 \%$ de la población elegida tenía EC, la mayoría de los participantes comunicó estar recibiendo estatinas como medicación habitual. Por lo tanto, es poco probable que una intervención sobre el estilo de vida modifique en forma notable los valores de LDL.

El uso de SMS en el ámbito de la salud puede ser incluido en lo que en los últimos años se definió como eHealth. El término incluye aplicaciones para teléfonos celulares inteligentes, SMS, historias clínicas electrónicas, herramientas para la toma de decisiones compartidas y todo aquello que fusione la salud con la tecnología.

Un avance en el campo de la investigación de estas intervenciones fue la publicación por parte del British Medical Journal (BMJ) de una guía para la evaluación del reporte de intervenciones que incluyen teléfonos celulares

\section{Conclusiones de la comentadora}

Con el advenimiento de la eHealth, se abrió un enorme campo de posibilidades para la creación de diferentes herramientas tecnológicas que integren al paciente al cuidado de su propia salud. ¿Será este el camino para mejorar la adherencia, empoderamiento y autogestión de los pacientes con enfermedades crónicas?

María Emilia Espósito [ Servicio de Medicina Familiar y Comunitaria del Hospital Italiano de BuenosAires. mariaemilia.esposito@ hospitalitaliano.org.ar ]

Espósito M. Una intervención basada en mensajes de texto fue útil para modificar factores de riesgo cardiovascular en pacientes con enfermedad coronaria. Evid Act Pract Ambul 2016;19(4):111. Comentado de: Chow C y col. Effect of Lifestyle-Focused Text Messaging on Risk Factor Modification in Patients With Coronary Heart Disease: A Randomized Clinical Trial .JAMA. 2015;314 (12):1255-63. PMID: 26393848.

\section{Referencias}

1. Franklin $\mathrm{V}$ y col. A randomized controlled trial of Sweet Talk, a text-messaging system to support young people with diabetes. Diabet Med. 2006;23 (12):1332-1338.

2. Márquez Contreras $\mathrm{E}$ y col. Effectiveness of an intervention to provide information to patients with hypertension as short text messages and reminders sent to their mobile phone (HTA-Alert)]. Aten Primaria. 2004;34 (8):399-405.

3. Eysenbach G. Whatis e-health?, J Med Internet Res 2001;3(2):e20.

4. Agarwal S. Guidelines for reporting of health interventions using mobile phones: mobile health (mHealth) evidence reporting and assessment (mERA) checklist, BMJ 2016;352:i1174 